\title{
INFLUÊNCIA DA TEMPERATURA E REGIME DE LUZ NA GERMINAÇÃO DE SEMENTES DE JANAGUBA (Himatanthus drasticus (Mart.) Plumel.) ${ }^{1}$
}

\author{
Influence of temperature and light on the germination of Janaguba \\ (Himatanthus drasticus (Mart.) Plumel.) seeds
}

\author{
Manoel Silva Amaro², Sebastião Medeiros Filho ${ }^{3}$, Renato Mendes Guimarães ${ }^{4}$, \\ Elizita Maria Teófilo ${ }^{5}$
}

\begin{abstract}
RESUMO
Com este trabalho, buscou-se determinar a influência da luz e da temperatura na germinação de sementes de janaguba. O ensaio foi conduzido no Laboratório de Análises de Sementes do Departamento de Fitotecnia, Universidade Federal do Ceará, Fortaleza-CE. Os tratamentos constaram de quatro temperaturas $\left(20^{\circ} \mathrm{C}, 25^{\circ} \mathrm{C}, 30^{\circ} \mathrm{C}\right.$ e $\left.20-35^{\circ} \mathrm{C}\right)$ e três condições de luz (escuro constante, fotoperíodo de $8 \mathrm{~h}$ luz e $16 \mathrm{~h}$ escuro e luz constante). Foram utilizadas 200 sementes por tratamento, semeadas sobre papel de filtro dispostos em placas de Petri, e acondicionadas em câmara de germinação por 20 dias, para determinação do percentual, velocidade e tempo médio de germinação. Utilizou-se o delineamento inteiramente casualizado, com 4 repetições de 50 sementes, em esquema fatorial 3 x 4 ( 3 condições de luz e 4 temperaturas). As médias foram comparadas pelo teste de Tukey, a 5\% de probabilidade. Houve diferença significativa na interação temperatura x luz, nas três variáveis. Concluiu-se que as sementes de janaguba apresentam fotoblastismo neutro; as temperaturas constantes de $20^{\circ} \mathrm{C} \mathrm{e} 25^{\circ} \mathrm{C}$, combinadas com escuro constante e luz/escuro, e as temperaturas constante de $30^{\circ} \mathrm{C}$ e alternada de $20-35^{\circ} \mathrm{C}$, combinadas com luz/escuro, são as condições mais favoráveis à germinação; a combinação das temperaturas constantes de $25^{\circ} \mathrm{C}$ e $30^{\circ} \mathrm{C}$ e ausência de luz, aumenta a velocidade e reduz o tempo médio de germinação de sementes de janaguba.
\end{abstract}

Termos para indexação: Planta medicinal, germinabilidade, propagação.

\begin{abstract}
This study aimed to evaluate the influence of the temperature and light on the germinative behaviour of janaguba (Himatanthus drasticus) seeds, with the experiments being conducted at the Laboratory of Seed Analysis of the Federal University of Ceará. To analyse percentage, velocity and average time of germination, the treatments consisted of four different temperatures $\left(20^{\circ} \mathrm{C}, 25^{\circ} \mathrm{C}\right.$, $30^{\circ} \mathrm{C} \mathrm{e} 20-35^{\circ} \mathrm{C}$ ) and three different light expositions (constant dark; $8 \mathrm{~h}$ of light plus $16 \mathrm{~h}$ of dark; and constant light). The treatments consisted of 200 seeds, with four repetitions of 50 seeds each, sown on a Germitest towel paper, displayed inside Petri dishes, and maintained for 20 days in germination chamber. A completely randomized $3 \times 4$ factorial design (three light expositions $\mathrm{x}$ four treatments) was used. The Tukey's Test at $5 \%$ of probability was used to compare the obtained averages. The janaguba seeds were shown to have neuter photoblastism. The better conditions for janaguma seed germination are temperatures of 20 and $25^{\circ} \mathrm{C}$ combined with constant dark and light/dark, constant temperature of $30^{\circ} \mathrm{C}$ and alternated temperature from 20 to $35^{\circ} \mathrm{C}$ combined with intermittent light/dark. The constant temperatures of 25 and $30^{\circ} \mathrm{C}$ plus constant dark led to the greatest indexes of velocity of germination and caused the reduction of the germination average time.
\end{abstract}

Index terms: Medicinal plant, viability, propagation.

(Recebido para a publicação em 27 de abril de 2004 e aprovado em 27 de outubro de 2005)

\section{INTRODUÇÃO}

Himatanthus drasticus é uma Apocynaceae da subfamília Plumeriodeae, distribuída geograficamente desde o Sudeste do Brasil até a Guiana Francesa, Suriname e Guiana. No Brasil, ocorre nos Estados de Minas Gerais, Bahia, Sergipe, Alagoas, Pernambuco, Rio Grande do Norte, Ceará, Paraíba, Piauí, Maranhão, Pará e Roraima. Além de designada, popularmente, como janaguba no Ceará, é conhecida como tiborna, jasmim-manga e raivosa em Minas Gerais e Bahia, pau-de-leite no Piauí, joanaguba no Rio Grande do Norte e sucuúba na Amazônia (PLUMEL, 1991).

Lorenzi \& Matos (2002), Modesto (1997) e Plumel (1991), destacam que a janaguba é uma espécie arbórea que pode atingir sete metros de altura, com folhagem densa nas extremidades dos ramos, folhas obovais, subcoriáceas, brilhantes, glabras, verde escuro, com ápice arredondado a obtuso e pecíolos curtos. Possui flores brancas, aromáticas, fruto tipo folículo, em forma de chifre, medindo

\footnotetext{
${ }^{1}$ Parte da Dissertação de Mestrado, defendida pelo primeiro autor, em Agronomia/Fitotecnia-UFC.

${ }^{2}$ Biólogo, Mestrado em Agronomia - Fitotecnia pela Universidade Federal do Ceará -Fortaleza, CE.

${ }^{3}$ Engenheiro Agrônomo, D.Sc., Professor do Departamento de Fitotecnia/CCA - Universidade Federal do Ceará/UFC - Fortaleza, CE - filho@ufc.br ${ }^{4}$ Engenheiro Agrônomo, D.Sc., Professor do Departamento de Agronomia da Universidade Federal de Lavras/UFLA - Cx. P. 3037 - $37.200-000$ Lavras, MG - renatomg@ufla.br

${ }^{5}$ Engenheira Agrônoma, D.Sc., Pesquisadora do Departamento de Fitotecnia - universidade Federal do Ceará/UFC - Fortaleza-CE - elizita@ufc.br
} 
entre 15 e $20 \mathrm{~cm}$ de comprimento por $2,5 \mathrm{~cm}$ de largura e sementes com alas concêntricas. A casca é rugosa e exsuda um látex branco bastante utilizado na medicina popular, principalmente pelos habitantes da região de ocorrência.

O processo germinativo de sementes constitui uma seqüência de eventos físicos, bioquímicos e fisiológicos, influenciados por vários fatores, que podem atuar isolados ou em interação. Um desses fatores é a temperatura, que altera a percentagem e velocidade de germinação, por agir na absorção de água pela semente e nas reações bioquímicas que regulam o metabolismo (BEWLEY \& BLACK, 1994; FIGLIOLIA et al., 1993).

Cada espécie possui uma temperatura adequada para sua germinação. Segundo Borges \& Rena (1993), e considerando a maioria das espécies tropicais, a temperatura ótima situa-se entre $20^{\circ} \mathrm{C}$ e $30^{\circ} \mathrm{C}$.

Berkenbrock \& Paulilo (1999), avaliando o efeito da presença e ausência de luz no processo germinativo de sementes de Maytenus robusta Reiss. e Hedyosmun brasiliense Mart., constataram germinação de sementes de $M$. robusta tanto em condições de luz como de escuro, além da ocorrência de maior crescimento inicial das plântulas sob maior intensidade de luz. No entanto, $H$. brasiliense comportou-se como fotoblástica positiva e com maior crescimento inicial das plântulas em condições de baixa luminosidade.

Apesar do recente interesse de vários pesquisadores sobre a propagação de espécies silvestres, ainda há carência de informações referentes às condições ideais de germinação. Isso pode ser verificado nas Regras para Análise de Sementes (BRASIL, 1992), nas quais são encontradas poucas prescrições para análise de sementes de espécies florestais.

Nesse aspecto, ainda são desconhecidas as exigências térmicas e luminosas para a germinação de sementes de janaguba,. Em consequiência, há prejuízos na avaliação da qualidade das sementes, principalmente quando se faz necessário comparar resultados obtidos em diferentes laboratórios.

O objetivou deste trabalho foi avaliar a influência da temperatura e da luz na germinação de sementes de janaguba.

\section{MATERIALE MÉTODOS}

Os frutos foram coletados manualmente, em setembro de 2001, de várias plantas, selecionadas ao acaso, na Floresta Nacional do Araripe, área de preservação ambiental, situada no altiplano da Chapada do Araripe, Crato-CE, com vegetação do tipo higrófila, média pluviométrica de $1.100 \mathrm{~mm} /$ ano e temperatura oscilante entre $18^{\circ} \mathrm{C}$ e $25^{\circ} \mathrm{C}$ (MODESTO, 1997) e relevo tabular com cotas mínimas de 840 e máximas de 920 metros.

As sementes foram extraídas de frutos maduros e embaladas em sacos plásticos e conduzidas ao Laboratório de Análise de Sementes do Departamento de Fitotecnia do Centro Ciências Agrárias, da Universidade Federal do Ceará, Fortaleza-CE, onde foi realizado o ensaio, no período de outubro de 2001 a janeiro de 2002.

Para as determinações da porcentagem, do índice de velocidade e do tempo médio de germinação, as sementes foram semeadas sobre dois discos de papel de filtro, dispostos em placas de Petri de $14 \mathrm{~cm}$ de diâmetro (50 sementes por placa), previamente umedecidos com água destilada na quantidade de 2,5 vezes a massa do papel seco, umedecendo-os, posteriormente, quando necessário. As placas de Petri foram acondicionadas em câmaras de germinação reguladas de forma a combinar quatro temperaturas $\left(20^{\circ} \mathrm{C}, 25^{\circ} \mathrm{C}, 30^{\circ} \mathrm{C}\right.$ e $\left.20-35^{\circ} \mathrm{C}\right)$ e três condições de luminosidade (escuro constante, luz constante e alternância de luz/escuro), constituindo 12 tratamentos.

$\mathrm{Na}$ alternância da temperatura estabeleceu-se $20^{\circ} \mathrm{C} /$ $16 \mathrm{~h}$ e $35^{\circ} \mathrm{C} / 8 \mathrm{~h}$ e no fotoperíodo 8 horas de luz a $35^{\circ} \mathrm{C}$ e 16 horas de escuro a $20^{\circ} \mathrm{C}$. A condição de escuro foi obtida pelo revestimento das placas de Petri com duas folhas pretas de plástico polietileno e as leituras foram realizadas na presença de luz verde de segurança, conforme recomendam Kendricks \& Frankneland (1983).

Para as três determinações foram utilizadas 200 sementes por tratamento, divididas em quatro repetições de 50. No teste de germinação a contagem foi feita aos 20 dias após a semeadura, considerando-se normais as plântulas que apresentavam radícula com comprimento igual ou superior a $5 \mathrm{~mm}$, sendo os resultados expressos em porcentagem.

$\mathrm{Na}$ determinação do índice de velocidade de germinação utilizou-se o material do teste de germinação (4 repetições de 50 sementes), realizando contagens diárias, durante 20 dias, das sementes germinadas com radículas de no mínimo $1 \mathrm{~mm}$ de comprimento. Para os cálculos adotou-se a fórmula proposta por Maguire (1962).

O tempo médio de germinação foi determinado utilizando-se o material do teste de germinação, tendo sido calculado utilizando a fórmula citada por Labouriau (1983), com o resultado expresso em dias após a semeadura.

Utilizou-se o delineamento inteiramente casualizado, em esquema fatorial 4 × $3 \mathrm{com} 4$ repetições, totalizando 12 tratamentos para testar os efeitos de quatro temperaturas $\left(20^{\circ} \mathrm{C}, 25^{\circ} \mathrm{C}, 30^{\circ} \mathrm{C}\right.$ e $20-35^{\circ} \mathrm{C}$ ) e três condições de luz (luz 
constante, luz/escuro alternados e escuro constante). Os dados obtidos foram submetidos à análise estatística conforme Banzatto \& Kronka (1992) e Gomes (1990) e as médias comparadas pelo teste de Tukey a $5 \%$ de probabilidade.

\section{RESULTADOS E DISCUSSÃO}

Houve diferença significativa tanto para os fatores temperatura e luz isolados como para sua interação, para as três variáveis: porcentagem, velocidade e tempo médio de germinação.

Na Tabela 1 são apresentados os valores médios dos dados da porcentagem de germinação. Pela interação significativa entre os fatores temperatura e luz, verifica-se que, para temperatura de $20^{\circ} \mathrm{C}$, as condições de escuro constante e alternância de luz/escuro propiciaram maior germinação. $\mathrm{Na}$ temperatura de $25^{\circ} \mathrm{C}$ não houve diferença na germinação quanto às condições de luz. Para as temperaturas fixa de $30^{\circ} \mathrm{C}$ e alternada de $20-35^{\circ} \mathrm{C}$ a condição de alternância luz/escuro destacou-se em relação aos demais regimes de luz. Nas condições de luz constante e alternância de luz/escuro não houve diferenças entre as temperaturas, mas, sob condição de luz/escuro observouse maior uniformidade no percentual de germinação; na condição escuro constante as temperaturas fixas $20^{\circ} \mathrm{C}$ e $25^{\circ} \mathrm{C}$ sobressaíram-se com melhor performance.

As sementes testadas sob as condições de laboratório não apresentaram problemas no tocante à germinação, pois o menor valor médio $(87 \%)$ pode ser considerado elevado, tratando-se de uma espécie silvestre.

Em estudos sobre a germinação de espécies florestais, Larcher (2000) recomendou o uso de temperaturas alternadas, já que essas condições simulam às do ambiente natural de florestas onde as flutuações de temperaturas ocorrem, principalmente, pela abertura de clareiras que estimulam a germinação de espécies pioneiras.

Em alguns casos a alternância de temperatura aumenta consideravelmente a germinação, como foi verificado em sementes de (Syzygium aromaticum (L.) Merr. \& Perry) e Dictyoloma vandellianum Adr. Juss. (BONACIN et al., 2001). Todavia, a alternância de temperatura pode inibir, parcialmente, o desenvolvimento do processo de germinação, a exemplo dos resultados encontrados por Posse et al. (2001), em sementes de pimentão (Capsicum annuum L.), e por Andrade \& Pereira (1994), em sementes de cedro (Cedrela odorata L.). Por outro lado, para algumas espécies, temperaturas constantes ou alternadas parecem não ter efeitos significativos na germinação de suas sementes. Bezerra et al. (2001), utilizando sementes de Melão-de-São Caetano (Momordica charantia L.), constataram ausência de efeitos de temperaturas constante de $20^{\circ} \mathrm{C}$ e alternada de $20-30^{\circ} \mathrm{C}$ na germinação.

Andrade et al. (2000), estudando o comportamento germinativo de sementes de jenipapo, conseguiram os melhores resultados com temperaturas fixas de $25 \mathrm{C}, 30^{\circ} \mathrm{C}$ e $35^{\circ} \mathrm{C}$.

Posse et al. (2001) constataram que sementes de pimentão germinam melhor em temperaturas fixa de $20^{\circ} \mathrm{C}$, $25^{\circ} \mathrm{C}$ e $30^{\circ} \mathrm{C}$.

TABELA 1 - Médias da porcentagem de germinação de sementes de janaguba submetidas à germinação em quatro temperaturas e três condições de luz. Fortaleza-CE, UFC, 2003.

\begin{tabular}{lccc}
\hline \multirow{2}{*}{ Temperatura } & \multicolumn{3}{c}{ Luz } \\
\cline { 2 - 4 } & Luz constante & Luzlescuro & Escuro constante \\
\hline $20^{\circ} \mathrm{C}$ & $92 \mathrm{bA}$ & $100 \mathrm{aA}$ & $100 \mathrm{aA}$ \\
$25^{\circ} \mathrm{C}$ & $94 \mathrm{a} \mathrm{A}$ & $97 \mathrm{aA}$ & $98 \mathrm{a} \mathrm{AB}$ \\
$30^{\circ} \mathrm{C}$ & $93 \mathrm{bA}$ & $99 \mathrm{aA}$ & $93 \mathrm{bBC}$ \\
$20-35^{\circ} \mathrm{C}$ & $88 \mathrm{bA}$ & $99 \mathrm{aA}$ & $87 \mathrm{bC}$ \\
\hline
\end{tabular}

$\mathrm{CV} \%=3,6$

Médias seguidas de mesma letra minúscula na linha e maiúscula na coluna, não diferem entre si pelo teste de Tukey a $5 \%$ de probabilidade.

Ciênc. agrotec., Lavras, v. 30, n. 3, p. 450-457, maio/jun., 2006 
$\mathrm{Na}$ condição de escuro contínuo, observou-se decréscimo da germinação em função da elevação da temperatura constante e do emprego de temperatura alternada, com extremos de $100 \%$ aos $20^{\circ} \mathrm{C}$ e $87 \%$ na temperatura $20-35^{\circ} \mathrm{C}$ (Tabela 1); o fato indica que as sementes de janaguba, sob a condição de escuro, têm sua germinação dependente da condição térmica. Foi verificado, também, que as sementes não germinadas apresentaramse visivelmente deterioradas, com sintomas principalmente de ataque de fungos.

Cassaro-Silva (2001), trabalhando com sementes de manduirana (Senna macranthera (Collad.) Irvin et Barn.), constatou um decréscimo de $83 \%$ na germinação quando a temperatura foi elevada de $27^{\circ} \mathrm{C}$ para $39^{\circ} \mathrm{C}$.

Larcher (2000) ressaltou ser ampla a faixa de temperatura para a germinação de sementes de espécies com ampla distribuição geográfica e daquelas adaptadas às grandes flutuações de temperaturas em seu habitat. Segundo esse autor a temperatura ótima para as espécies tropicais situa-se entre $20^{\circ} \mathrm{C}$ e $35^{\circ} \mathrm{C}$ em condições naturais; a combinação de luz e escuro com temperaturas alternadas pode propiciar maior uniformidade na germinação, já que aproximaria-se das condições do ambiente natural das espécies. Por outro lado, o autor afirmou que, até certos limites, o aumento de temperatura pode promover mudanças no desempenho de certas enzimas que atuam nos processos bioquímicos da germinação, além de propiciar a contaminação por microrganismos. Popinigis (1985) referiu-se ao efeito das temperaturas elevadas no aumento da atividade respiratória e proliferação de fungos

Carvalho \& Nakagawa (2000) afirmaram que na germinação, além do aumento no ataque de microrganismos, temperaturas acima de um determinado limite considerado ótimo para o total de germinação de uma dada espécie aceleram a velocidade dos processos bioquímicos da respiração, porém, propicia uma desorganização dos mesmos, de sorte que o número de sementes que consegue completá-lo vai diminuindo. Portanto, de acordo com as considerações dos autores, e levando-se em conta o número de sementes atacadas por fungos, esse decréscimo na germinação em função do aumento de temperatura pode ser interpretado como uma deterioração de algumas sementes pelo ataque de microrganismos.

Em relação às condições de luminosidade, pode-se observar pelos dados, que para as sementes de janaguba, os tratamentos luz/escuro, em qualquer temperatura, e escuro constante a $20^{\circ} \mathrm{C}$ e $25^{\circ} \mathrm{C}$ como os de melhor performance em relação aos demais. Nesse sentido, pode-se dizer que as sementes de janaguba são insensíveis à luz visto a germinação ter ocorrido tanto na luz como no escuro.

A inclusão de uma semente na categoria das sensíveis ou insensíveis à luz depende das condições de maturação, armazenamento, temperatura de embebição e incubação e tratamento osmótico (LADEIRA et al., 1987).

A indiferença à luz na germinação das sementes refere-se a um comportamento comumente descrito para árvores de sub-bosque e plantas de sombra (ANDRADE, 1995); contudo, Himatanthus drasticus parece ter comportamento heliofítico, conforme as descrições de Lorenzi (1998) para espécies do gênero Himatanthus.

Insensibilidade de sementes na germinação em relação à luz também foi demonstrada nos resultados de Berkenbrock \& Paulilo (1999), em sementes de Maytenus robusta; França et al. (2002), com sementes de batata-depurga amarela (Operculina alata (Ham.) Urban) e de Perez et al. (2001), testando sementes de canafístula (Peltophorum dubium (Spreng.) Taub.)

As altas porcentagens de germinação nas condições de luz/escuro e escuro constante a $20^{\circ} \mathrm{C}$, atingindo $100 \%$, bem como a maior uniformidade da germinação na alternância de luz/escuro sob qualquer temperatura, pode estar relacionada com as oscilações de temperatura e alternância de luz comumente verificadas no habitat natural da espécie (LARCHER, 2000).

Amaro (2002) verificou que a janaguba apresenta característica comumente encontrada em espécies pioneiras, nas quais a germinação depende, geralmente, da luz para ocorrer. O autor constatou na Floresta Nacional do Araripe, onde há maior sombreamento, dificuldades de crescimento da espécie haja vista que, em uma média de 53 plantas.ha-1 ${ }^{-1}$ não foram encontradas plantas jovens. Todavia, no área de Cerradão, onde a vegetação é mais aberta, com ocorrência de grandes clareiras, de uma média 616 plantas.ha ${ }^{-1}, 60,5 \%$ foram plantas jovens. No entanto, as análises estatísticas demonstraram que as sementes obtiveram uma maior germinação na alternância de luz e escuro e na ausência de luz, a $20^{\circ} \mathrm{C}$, característica comum encontrada em espécies de vegetação secundária.

A germinação de espécies pioneiras pode apresentar insensibilidade à luz, a exemplo dos resultados obtidos por Perez et al. (2001), em sementes de canafístula, espécie considerada heliófita e pioneira por Lorenzi (1998). Por outro lado, espécies de vegetação secundária apresentam a germinação dependente de luz, como ocorre em Coccocypselum guianenses (Aubl.) K. Shum., uma espécie de estágios sucessionais mais avançados, que apresenta fotoblastismo positivo (GUIMARÃES \& QUEIROZ, 1997, 
1998). Conforme Berkenbrock \& Paulilo (1999), o requerimento de luz para germinar parece ser um fenômeno associado a sementes pequenas, possuidoras de pouco material de reserva, o que não é o caso das sementes de janaguba.

$\mathrm{Na}$ Tabela 2, são dispostos os dados médios do indice de velocidade de germinação obtidos nos diferentes tratamentos de temperaturas e regimes de luz. Para todas as condições de temperatura, o escuro constante proporcionou maior índice de velocidade de germinação.

Quanto às condições de luz ou escuro constantes, as temperaturas de $25^{\circ} \mathrm{C}$ e $30^{\circ} \mathrm{C}$ foram as que propiciaram índices mais elevados de velocidade de germinação. Estes resultados estão em concordância com àqueles obtidos por França et al. (2002), em sementes de batata-de-purga amarela, quando a combinação de $25^{\circ} \mathrm{C}$ com o escuro destacou-se quanto à velocidade do processo de germinação. No entanto, Silva et al. (2002) observaram, em sementes de cambará (Vochysia haenkiana Mart.), maior índice de velocidade de germinação sob a temperatura fixa de $20^{\circ} \mathrm{C}$, tanto na presença como na ausência de luz. Almeida et al. (2001) constataram que essa mesma temperatura, combinada com luz, foi a condição ambiental que proporcionou maior velocidade de germinação em sementes de Leonorus sibiricus L.

Já Souza et al. (2001) verificaram em Plantagro ovata L., ausência de diferença significativa no índice de velocidade de germinação quando as sementes foram expostas a $20^{\circ} \mathrm{C}$ em condições de luz ou escuro. CassaroSilva (2001) obteve maior velocidade na germinação de sementes de manduirana nas temperaturas constantes de $24^{\circ} \mathrm{C}, 27^{\circ} \mathrm{C}$ e $30^{\circ} \mathrm{C}$ com alternância de luz e escuro.

Constatou-se, ainda, conforme a Tabela 2 que o aumento nas temperaturas constantes promoveu elevação da velocidade de germinação, nas três condições de luz, enquanto que na temperatura alternada $20-35^{\circ} \mathrm{C}$, sob os três regimes luminosos, houve redução na velocidade de germinação. Acréscimo na velocidade germinação em função do aumento da temperatura também foi constatado por Andrade (1995) em sementes de Tibouchina benhtamiana Cogn., Tibouchina grandifolia Cogn. e Tibouchina moricandiana Baill., mantidas sob $8 \mathrm{~h}$ de luz e $16 \mathrm{~h}$ de escuro.

Zhao et al. (1994) referiram-se à aceleração da atividade respiratória como consequiência da elevação da temperatura, resultando no aumento da velocidade de germinação; porém, em algumas espécies, o aumento térmico pode causar redução do número total de sementes germinadas. Em contrapartida, conforme Larcher (2000), baixas temperaturas podem reduzir as taxas metabólicas e, conseqüentemente, diminuir a velocidade de germinação e o número de sementes germinadas no final do teste.

Os valores referentes ao tempo médio de germinação estão apresentados na Tabela 3. Observou-se, para as temperaturas fixas de $20^{\circ} \mathrm{C}, 25^{\circ} \mathrm{C}$ e $30^{\circ} \mathrm{C}$, que a condição de escuro constante promoveu redução do tempo médio de germinação; na temperatura alternada de $20-35^{\circ} \mathrm{C}$, menores tempos foram observados quando as sementes foram germinadas sob luz constante ou escuro constante.

Para as condições de luz, constatou-se que sob regimes de luz e escuro constantes os menores valores do tempo médio de germinação ocorreram nas temperaturas fixas de $25^{\circ} \mathrm{C}, 30^{\circ} \mathrm{C}$ e alternada de $20-35^{\circ} \mathrm{C}$, enquanto que na condição de luz/escuro destacou-se a temperatura fixa de $30^{\circ} \mathrm{C}$, com menor tempo médio de germinação.

Ao se confrontar os valores do tempo médio de germinação com os da velocidade de germinação constatouse que, para a maioria dos tratamentos, quanto maior foi o índice de velocidade de germinação menor o tempo médio de germinação.

TABELA 2 - Médias do índice de velocidade de germinação de sementes de janaguba submetidas à germinação em quatro temperaturas e três condições de luz. Fortaleza-CE, UFC, 2003.

\begin{tabular}{lccc}
\hline \multirow{2}{*}{ Temperatura } & \multicolumn{3}{c}{ Luz } \\
\cline { 2 - 4 } & Luz constante & Luzlescuro & Escuro constante \\
\hline $20^{\circ} \mathrm{C}$ & $3,50 \mathrm{Bbc}$ & $3,51 \mathrm{Bab}$ & $4,50 \mathrm{Ab}$ \\
$25^{\circ} \mathrm{C}$ & $4,30 \mathrm{Bab}$ & $3,84 \mathrm{Bab}$ & $5,49 \mathrm{Aa}$ \\
$30^{\circ} \mathrm{C}$ & $4,68 \mathrm{Ba}$ & $4,01 \mathrm{Ba}$ & $5,75 \mathrm{Aa}$ \\
$20-35^{\circ} \mathrm{C}$ & $3,48 \mathrm{Bc}$ & $3,21 \mathrm{Bb}$ & $4,59 \mathrm{Ab}$ \\
\hline
\end{tabular}

$\mathrm{CV} \%=9,0$

Médias seguidas de mesma letra minúscula na coluna e maiúscula na linha, não diferem entre si pelo teste de Tukey a $5 \%$ de probabilidade.

Ciênc. agrotec., Lavras, v. 30, n. 3, p. 450-457, maio/jun., 2006 
TABELA 3 - Médias do tempo médio de germinação (em dias) de sementes de janaguba submetidas à germinação em quatro temperaturas e três condições de luz. Fortaleza-CE, UFC, 2003.

\begin{tabular}{lccc}
\hline \multirow{2}{*}{ Temperatura } & \multicolumn{3}{c}{ Luz } \\
\cline { 2 - 4 } & Luz constante & Luz/escuro & Escuro constante \\
\hline $20^{\circ} \mathrm{C}$ & $13,5 \mathrm{aA}$ & $13,1 \mathrm{aB}$ & $11,2 \mathrm{bA}$ \\
$25^{\circ} \mathrm{C}$ & $11,6 \mathrm{aAB}$ & $12,1 \mathrm{aB}$ & $8,5 \mathrm{bB}$ \\
$30^{\circ} \mathrm{C}$ & $10,3 \mathrm{abB}$ & $10,5 \mathrm{aC}$ & $8,5 \mathrm{bB}$ \\
$20-35^{\circ} \mathrm{C}$ & $11,8 \mathrm{bB}$ & $15,7 \mathrm{aA}$ & $10,1 \mathrm{bAB}$ \\
\hline
\end{tabular}

$\mathrm{CV} \%=9,9$

Médias seguidas de mesma letra minúscula na linha e maiúscula na coluna, não diferem entre si pelo teste de Tukey a $5 \%$ de probabilidade.

Comparando-se a porcentagem de germinação (Tabela 1) com o índice de velocidade de germinação (Tabela 2), verificou-se que os maiores percentuais de germinação ocorreram nos tratamentos luz alternada, em todas as temperaturas, e na ausência de luz nas temperaturas de $20^{\circ} \mathrm{C}$ e $25^{\circ} \mathrm{C}$. Por outro lado, os maiores índices de velocidade de germinação ocorreram no escuro nas temperaturas fixas de $25^{\circ} \mathrm{C}$ e $30^{\circ} \mathrm{C}$.

Estes resultados concordam com Carvalho \& Nakagawa (2000) e Popinigis (1985), ao ressaltarem que o maior índice de velocidade de germinação não implica em maior porcentagem de germinação ou maior número de sementes germinadas ao final do teste.

\section{CONCLUSÕES}

As sementes de janaguba apresentam fotoblastismo neutro;

As temperaturas constantes de $20^{\circ} \mathrm{C}$ e $25^{\circ} \mathrm{C}$, combinadas com escuro constante e luz/escuro, e as temperaturas constante de $30^{\circ} \mathrm{C}$ e alternada de $20-35^{\circ} \mathrm{C}$, combinadas com a alternância de luz/escuro, são as condições mais favoráveis à germinação;

A combinação das temperaturas constantes e $25^{\circ} \mathrm{C}$ e $30^{\circ} \mathrm{C}$ ausência de luz promovem maiores índices de velocidade de germinação e redução do tempo médio de germinação de sementes de janaguba.

\section{REFERÊNCIAS BIBLIOGRÁFICAS}

ALMEIDA, L. F. R.; POLETTO, R. S.; SOUSA, M. P.; BRAGA, J. F.; DELACHIAVE, M. E. A. Temperatura e luz na germinação de sementes de Leonorus sibiricus L. In: JORNADA PAULISTA DE PLANTAS MEDICINAIS, 5., 2001, Botucatu. Anais... Botucatu: Unesp, 2001. p. 118.
AMARO, M. S. Extração, uso e aplicações do látex de janaguba na Região do Cariri e suas conseqüências na Chapada do Araripe. 2002. 50 f. Monografia (Especialização em Ecologia) - Universidade Regional do Cariri, Crato, 2002.

ANDRADE, A. C. S. Efeito da luz e da temperatura na germinação de Leandra breviflora Cogn., Tibouchina benthamiana Cogn., Tibouchina grandifolia Cogn. e Tibouchina moricandiana (DC.) BAILL. (MELASTOMATACEAE). Revista Brasileira de Sementes, Brasília, v. 17, n. 1, p. 29-35, 1995.

ANDRADE, A. C. S.; SOUZA, A. F.; RAMOS, F. N.; PEREIRA, T. S.; CRUZ, A. P. M. Germinação de sementes de jenipapo: temperatura, substrato e morfologia do desenvolvimento pós-seminal. Pesquisa Agropecuária Brasileira, Brasília, v. 35, n. 3, p. 609-615, mar. 2000.

ANDRADE, C. S.; PEREIRA, T. S. Efeito do substrato e da temperatura na germinação e no vigor de sementes de cedro (Cedrela odorata L.-Meliaceae). Revista Brasileira de Sementes, Brasília, v. 16, n. 1, p. 34-40, 1994.

BANZATTO, D. A.; KRONKA, S. N. Experimentação agrícola. 2. ed. Jaboticabal: UNESP, 1992. 247 p.

BERKENBROCK, I. S.; PAULILO, M. T. S. Efeito da luz na germinação e no crescimento inicial de Maytenus robusta Reiss. e Hedyosmum brasiliense Mart. Revista Brasileira de Sementes, Brasília, v. 21 n. 2, p. 243-248, 1999.

BEWLEY, J. D.; BLACK, M. Seeds: physiology of devolopment and germination. New York: Plenum, 1994. $445 \mathrm{p}$. 
BEZERRA, A. M. E.; MOMENTÉ, V. G.; CHAVES, F. C. M. Germinação de dois tipos de melão-de-São Caetano em três regimes de temperatura. In: JORNADA PAULISTA DE PLANTAS MEDICINAIS, 5., 2001, Botucatu. Anais... Botucatu: UNESP, 2001. p. 120.

BONACIN, G. A.; ALMEIDA, J. B. S. A.; SANTOS, S. R. G.; PIVETTA, K. F. L. Germinação de sementes de Dictiyoloma vandellianum Adr. Juss. (tingui) em diferentes temperaturas e substratos. In: CONGRESSO BRASILEIRO DE FISIOLOGIA VEGETAL, 8., 2001, Ilhéus. Anais... Ilhéus: [s.n.], 2001. CD-ROM.

BORGES, E. E. L.; RENA, A. B. Germinação de sementes. In: AGUIAR, I. B.; PIÑA-RODRIGUES, F. C. M.; FIGLIOLIA, M. B. Sementes florestais tropicais. Brasília, DF: ABRATES, 1993. p. 83-135.

BRASIL. Ministério da Agricultura e Reforma Agrária. Regras para análise de sementes. Brasília, DF: SNDA/ DNDV/CLAV, 1992. 362 p.

CARVALHO, N. M.; NAKAGAWA, J. Sementes: ciência, tecnologia e produção. 4. ed. Jaboticabal: FUNEP, 2000. $588 \mathrm{p}$.

CASSARO-SILVA, M. Efeito da temperatura na germinação de sementes de manduirana (Senna macranthera (Collad.) Irwin et Barn.-CAESALPINIACEAE). Revista Brasileira de Sementes, Brasília, v. 23, n. 1, p. 92-99, 2001.

FIGLIOLIA, M. B.; OLIVEIRA, E. C.; PIÑA-RODRIGUES, F. C. M. Análise de sementes. In: AGUIAR, I. B.; PIÑARODRIGUES, F. C. M.; FIGLIOLIA, M. B. Sementes florestais tropicais. Brasília, DF: ABRATES, 1993. p. 137174.

FRANÇA, E. A.; MEDEIROS-FILHO, S.; FREITAS, J. B. S. Avaliação da germinação de sementes de batata-de-purga amarela em dois substratos e cinco condições ambientais. Ciência e Agrotecnologia, Lavras, v. 26, p. 232-236, mar./ abr. 2002.

GOMES, F. P. Curso de estatística experimental. 13. ed. Piracicaba: ESALQ, 1990. 468 p.

GUIMARÃES, F. B.; QUEIROZ, M. H. Fotoblastismo como estratégia germinativa de diferentes grupos ecológicos da floresta Ombrófila Densa. Informativo Abrates, Curitiba, v. 7, n. 1, p. 234, 1997.

GUIMARÃES, F. B.; QUEIROZ, M. H. Frutos, sementes e germinação de Coccocypselum canascens Willd. e Coccocypselum guianenses (Aubl.) K. Shum. Rubiaceae. In: CONGRESSO NACIONAL DE BOTÂNICA, 49., 1998, Salvador. Resumos... Salvador: UFB, 1998. p. 190.

KENDRICKS, R. E.; FRANKNELAND, B. Phitocrome and plant. In: ARNOLD, E. (Ed.). Southamptobn. [S.l.]: Camelot, 1983. $76 \mathrm{p}$.

LABOURIAU, L. G. A germinação de sementes. Washington: Secretaria Geral da Organização dos Estados Americanos, 1983. 173 p.

LADEIRA, A. M.; GUARDIA, M. C.; TAKAKI, M. Manipulation of seede germination in Plantago tomentosa Lant. and Rhapanus sativus. Seed Science and Tecnology, Zurich, v. 15, p. 55-63, 1987.

LARCHER, W. Ecofisiologia vegetal. São Carlos: Rima, 2000. $531 \mathrm{p}$.

LORENZI, H. Árvores brasileiras: manual de identificação e cultivo de plantas arbóreas nativas do Brasil. Nova Odessa: Plantarun, 1998. 222 p.

LORENZI, H.; MATOS, F. J. A. Plantas medicinais no Brasil: nativas e exóticas. Nova Odessa: Plantarun, 2002. $427 \mathrm{p}$.

MAGUIRRE, J. D. Speed of germination-aid in selection and evaluation for seedling emergence and vigor. Crop Science, Madison, v. 2, p. 176-177, 1962.

MODESTO, M. M. L. S. Aspectos ecológicos e sócioeconômicos de Himatanthus articulata (Wahl.) Woodson. “janaguba" da Chapada doAraripe. 1997. 55 f. Monografia (Especialização em Botânica) - Universidade Regional do Cariri, Crato, 1997.

PEREZ, S. C. J. G. A.; FANTI, S. C.; CASALI, C. A. Influência da luz na germinação de sementes de canafístula submetidas ao estresses hídrico. Bragantia, Campinas, v. 3 , n. 60, p. 155-166, 2001.

Ciênc. agrotec., Lavras, v. 30, n. 3, p. 450-457, maio/jun., 2006 
PLUMEL, M. M. Le genre Himatanthus (Apocinaceae) revisión taxonomique: bradea. Boletim do Herbarium Bradeanu, Rio de Janeiro, v. 5, p. 1-20, 1991.

POPINIGIS, F. Fisiologia da semente. 2. ed. Brasília, DF: AGIPLAN, 1985. $289 \mathrm{p}$.

POSSE, S. C. P.; SILVA, R. F.; VIEIRA, H. D.; CATUNDA, P. H. A. Efeito do condicionamento osmótico e da hidratação na germinação de sementes de pimentão (Capsicum annиит L.). Revista Brasileira de Sementes, Brasília, v. 23, n. 1, p. 123-127, 2001.

SILVA, V. P.; COSTA, R. B.; NOGUEIRA, A. C.; ALBRECHT, J. M.; ARAÚJO, A. J. Influência da temperatura e luz na germinação de sementes de cambará (Vochysia haenkiana,
Mart.). Agrotropical, Cuiabá, v. 4, 2002. Disponível em: <http//ufmt.br./agrotrop/Revista4/doc/07\%20.htm>. Acesso em: 22 nov. 2002.

SOUZA, M. P.; BRAGA, L. F.; BRAGA, J. F.; DELACHIAVER, M. E. A.; SANTOS, F. A. Temperatura e fotoblastismo na germinação de sementes de Plantago ovata L. In: JORNADA PAULISTA DE PLANTAS MEDICINAIS, 5., 2001, Botucatu. Anais... Botucatu: UNESP, 2001. p. 121.

ZHAO, Y. T.; CHANG, R. Z.; LI, Y. J.; LIANG, B. W.; SUN, J. Y. Relationship between physical characters, chemical composition, germination and cold-tolerance in soybean. Chinese Journal of Botany, China, v. 6, n. 1, p. 60-63, 1994. 